\title{
DISPOSITIVOS $O$ SERVICIOS TECNOLÓGICOS DURANTE LA PRÁCTICA DE ACTIVIDAD FÍSICA PARA PERSONAS CON ENFERMEDAD: REVISIÓN SISTEMÁTICA'
}

\author{
García Belloso Amara \\ amgarciab@alumnos.unex.es \\ Macías Leal María Dolores \\ mamacias|@alumnos.unex.es \\ Facultad de Educación de la Universidad de Extremadura
}

Recepción artículo: 27 octubre 2021

Admisión Evaluación: 27 octubre 2021

Informe Evaluador 1: 12 noviembre 2021

Informe Evaluador 2: 12 noviembre 2021

Aprobación Publicación: 25 noviembre 2021

\section{RESUMEN}

Gracias a los avances tecnológicos se han elaborado diversos servicios web que fomentan la actividad física a personas con diversas enfermedades.

Objetivo: Conocer los tipos de dispositivos o servicios basados en la tecnología que se han desarrollado para ayudar a los usuarios con enfermedades con el objetivo de mantener y crear rutinas relacionadas con la actividad física o deporte. Así como conocer las diferentes enfermedades que se suelen tratar con este tipo de tecnología.

Método: La presente revisión sistemática, realiza un método descriptivo como forma de extracción de la información mediante un análisis profundo y detallado sobre la información recogida, seleccionando una serie de variables y explicando sus frecuencias para desarrollar así la realidad con la que nos encontramos.

Resultado: De los artículos seleccionados el 35\% de los artículos pertenecen a Estados Unidos; la mayoría de ellos son de tipo cuantitativo y la edad de la muestra que más predomina son mayores de 18 años, una población adulta. La enfermedad que predomina en cuanto al estudio del servicio web es la diabetes tipo 2 siendo la diabetes tipo 1 la segunda más estudiada y la depresión la que continúa.

Conclusiones: La producción cientíitica ha aumentado a lo largo de los años en relación con las enfermedades actuales, la enfermedad más trabajada es la diabetes de tipo 2 y las aplicaciones son el tipo de servicio web que más abunda en la actualidad.

Palabras clave: actividad física, servicios web y enfermedades.

\section{ABSTRACT}

Introduction: Thanks to technological advances, various web services have been developed that promote physical activity for people with various diseases. 


\section{DISPOSITIVOS O SERVICIOS TECNOLÓGICOS DURANTE LA PRÁCTICA DE ACTIVIDAD FÍSICA PARA PERSONAS CON ENFERMEDAD: REVISIÓN SISTEMÁTICA}

Objective: To understand the types of technology-based devices or services that have been developed to help users with illnesses with the aim of maintaining and creating physical activity or sport-related routines. As well as knowing the different diseases that are usually treated with this type of technology.

Method: This systematic review, performs a descriptive method as a way of extracting the information through a deep and detailed analysis of the information collected, selecting a series of variables and explaining their frequencies in order to develop the reality with which we find ourselves.

Result: Of the selected articles, $35 \%$ of the articles belong to the United States; most of them are quantitative and the age of the most predominant sample is over 18 years old, an adult population. The disease that predominates in terms of the study of the web service is type 2 diabetes, being type 1 diabetes the second most studied and depression the one that continues.

Conclusions: Scientific output has increased over the years in relation to current diseases, the most worked disease is type 2 diabetes and applications are the most abundant type of web service today.

Keywords: physical activity, web services and diseases

\section{INTRODUCCIÓN}

De acuerdo con Muñoz, Garrote y Sánchez (2017), el deporte en las personas con discapacidad cada vez son más analizadas debido a la evidencia de los beneficios que aportan en su vida.

En la actualidad las personas cada vez están más familiarizadas con los dispositivos inteligentes, de tal forma que puede aplicarse con fines educativos. Así pues, diversas investigaciones estudian la manera de utilizar la tecnología para gestionar y realizar cambios de comportamientos, como los relacionados con la intervención de actividad física, dejar de fumar, hábitos alimenticios, etc.

En este sentido, la tecnología móvil, los programas basados en internet (como plataformas online, foros, etc.), relojes inteligentes, tecnologías de asistencia, portátil, televisión, e-books y otros dispositivos se han aplicado en numerosas intervenciones para el cambio de conducta de salud, sobre todo en adultos.

De acuerdo con Gabrielli et. al (2017), las personas han comenzado a desarrollar un fuerte apego a sus dispositivos móviles y mantienen con frecuencia esta tecnología con ellos todo el tiempo. Por esta razón, el desarrollo de nuevos servicios (aplicaciones, programas webs...) o dispositivos tecnológicos durante la práctica deportiva para personas con discapacidad o enfermedad supone un reto en la actualidad, como forma de motivar, involucrar y fomentar la actividad física.

Es innegable la importancia de la participación de las personas con alguna enfermedad en actividades físico-deportivas, así como la adopción de estilos de vida más saludables. Por lo tanto, nos planteamos la siguiente pregunta: ¿Qué tipos de dispositivos web existen en la actualidad que fomenten la actividad física a personas con enfermedades?

Según Prieto (2011), la actividad física son todas aquellas actividades que realizamos a lo largo del día en las cuales consumimos energía a través del movimiento corporal (andar, limpiar, bailar, jugar, etc.). A diferencia del ejercicio físico, el cual se realiza de forma intencionada, planificada y organizada, cuyo objetivo es mantener o mejorar la forma física.

Al ingresar a la Web, se ingresa a un espacio virtual, distinto del real pero que tiene gran incidencia sobre él. Uno de los usos más extendidos de internet tiene relación con el ocio y el tiempo libre. Muchas personas, en especial los jóvenes, utilizan internet para interactuar en las redes sociales como Facebook y Twitter de ahí la importancia de utilizarlas como medios para informar sobre la actividad física y tratar de mejorar la salud (Cantúa, Espinoza, Figueroa, Romero, Ruiz, Andrade, 2018).

Álvarez (2020), sostiene que una de las áreas de prevención sanitaria que más importancia están ocupando, es la relacionada con enfermedades que tiene que ver con el sedentarismo, falta de ejercicio, diabetes o dietas poco equilibradas y que, afortunadamente, las tecnologías inteligentes han abarcado estas áreas de la salud, desarrollando herramientas informáticas que facilitan el diagnóstico y tratamiento de las enfermedades en todos los niveles en los últimos años. 
Es importante tener en cuenta que, como nos explica Montaña, Amado y Eslava (2014), Ios sistemas de comunicación y las grandes redes de telemedicina van dejando de ser tema de países desarrollados al convertirse en una necesidad para una vida digna, sobre todo si se vive en lugares alejados, ofreciendo servicios y atenciones oportunas; En zonas rurales se ha logrado algún éxito en la mejora del acceso a la asistencia sanitaria gracias a la oportunidad que representa la tecnología avanzada y los teléfonos móviles.

Las nuevas tecnologías han tomado un papel muy relevante en nuestras vidas, siendo las principales fuentes de búsqueda de información y comunicación. Por ello, y el relación con la definición anteriormente descrita, tal y como nos indica Muñoz, Villada y Giraldo (2013), la inserción de estas nuevas tecnologías en ambientes donde se realiza la AF permite además, dinamizar la relación entre el ejercicio y los resultados, fusionando una necesidad general del común como lo es la estimulación y motivación para realizar la AF solución innovadora que plantea roles de jugabilidad en donde se utilizan micro objetivos que pueden ser alcanzados por el usuario, brindando entretenimiento y experiencias satisfactorias en beneficio de la salud del mismo.

Sin embargo, es importante diferenciar diversos servicios web trabajados en este estudio. Por ello, cabe destacar que una aplicación móvil es un programa que se descarga e instala en el dispositivo móvil de un usuario, mientras que un sitio web para móviles no es más que una página web adaptada a los formatos de tabletas y teléfonos inteligentes. Por otro lado, se puede confundir también el concepto de aplicaciones y programas. En este sentido, la diferencia radica en que los programas están diseñados para funcionar en una plataforma única, mientras que las aplicaciones se desarrollan para funcionar en más de una plataforma.

Así pues, el objetivo principal es realizar una revisión sistemática para conocer los dispositivos o servicios basados en la tecnología que se han desarrollado para ayudar a los usuarios con enfermedades con el objetivo de mantener y crear rutinas relacionadas con la actividad física o deporte. Así como conocer las diferentes enfermedades que se suelen tratar con este tipo de tecnología.

Las hipótesis planteadas a la que se intenta dar respuesta son las siguientes: la producción científica ha aumentado a lo largo de los años en relación con las enfermedades actuales; la obesidad es la enfermedad más trabajada en cuanto a los servicios web creados en la actualidad; las aplicaciones para enfermedades son el tipo de servicios web que más abunda en la actualidad.

\section{MÉTODO}

\section{Procedimiento de estrategia de búsqueda: bases de datos, criterios de inclusión y proceso de selección.}

El presente artículo es una revisión sistemática de artículos científicos, en el cual, se recopiló información sobre los dispositivos o servicios tecnológicos durante la práctica de actividad física dirigida a personas con alguna enfermedad, publicados en diferentes bases de datos y siguiendo un diseño preestablecido. Este estudio, realiza un método descriptivo como forma de extracción de la información mediante un análisis profundo y detallado sobre la información recogida, seleccionando una serie de variables y explicando sus frecuencias para desarrollar así la realidad con la que nos encontramos.

El tema elegido se originó debido al interés personal común entre las integrantes del grupo, donde surgió la pregunta de investigación: ¿Existen dispositivos o aplicaciones creadas especialmente para personas con alguna enfermedad?

La búsqueda se realizó en diferentes bases de datos como: WOS, Google Scholar, Scopus y Pubmed. La mayoría de las bases de datos son gratuitas, no obstante, gracias al acceso que ofrece la UEX, se ha podido realizar la búsqueda en WOS. Asimismo, el periodo de búsqueda de los artículos seleccionados se realizó durante dos semanas, desde el 22 de marzo hasta el 5 de abril del 2021. La estrategia de búsqueda empleada ha sido similar en las 4 bases de datos, usando descriptores como: dispositivos o servicios basados en la tecnología, enfermedad y actividad física o deporte. Utilizando estas palabras clave, se ha usado los booleanos and y or. Un ejemplo de la estrategia de búsqueda es la que se muestra a continuación utilizada en la base de datos WOS: "Technology-based devices" OR "technology-based services" AND illness AND "physical activity" OR sport. 


\section{DISPOSITIVOS O SERVICIOS TECNOLÓGICOS DURANTE LA PRÁCTICA DE ACTIVIDAD FÍSICA PARA PERSONAS CON ENFERMEDAD: REVISIÓN SISTEMÁTICA}

La selección de los estudios que se ha obtenido finalmente, fueron a través de unos criterios de inclusión acordados al principio del estudio. El principal criterio de inclusión fue que los artículos estuvieran publicados a partir del año 2013 con datos relevantes sobre nuestro tema escogido. Otros criterios de inclusión fueron el idioma, (artículos publicados en inglés, independientemente del país donde se publicó) del área temática sobre Ciencias Sociales y de acceso abierto o disponibles en las bases de datos accesibles a estudiantes de la UEX. Si existía un desacuerdo entre las integrantes del trabajo con respecto a la inclusión de determinados artículos, se discutía hasta llegar a un consenso. Además, se incluyen los estudios que tras pasar los criterios de calidad a través del método Prisma obtienen más de un 49\% de puntuación.

Por otro lado, los criterios de exclusión fueron los artículos que tras pasar los criterios de calidad no superan cada uno el 49\% de los ítems establecidos. Se excluyeron también los artículos los cuales no cumplían con el objetivo propuesto para el estudio. Además, también se excluyeron aquellos artículos que se encontraban duplicados, aquellos que no estaban en acceso abierto, artículos en los cuales el texto no se encontraba completo, el título no era de nuestro interés y el idioma, en nuestro caso solamente texto en inglés. Una vez realizado el proceso de exclusión por conveniencia, se pasa a la extracción de datos.

\section{Extracción de datos y calidad de estudios}

Los datos de estudio fueron extraídos por las autoras manualmente, utilizando hojas de cálculo de Excel a través de la plataforma DRIVE para trabajar conjuntamente desde los ordenadores personales. Las variables que sirven para la realización del estudio fueron: el tipo de enfermedad y el tipo de servicio web, los cuales son variables nominales. Como variables de cadena, se extraen los autores, el título, ejemplos citados, los hallazgos 0 conclusiones clave, los beneficios, las barreras y obstáculos de uso y las necesidades o sugerencias de mejora. Y finalmente, las variables numéricas que se han recogido son el año de publicación, el porcentaje de calidad y los ítems cumplidos en los criterios de calidad.

Para evaluar la calidad de los artículos seleccionados se ha seguido los criterios de calidad de Law, M., et al (1998). De los 40 artículos seleccionados, tras pasar dichos criterios de calidad finalmente 37 artículos cumplen el 50\% de los ítems seleccionados. Los cuales oscilan entre el $56,24 \%$ al $100 \%$ de calidad.

Los 3 artículos restantes se han excluido tras no superar al menos el 49\% de los criterios de calidad individualmente.

El método utilizado para evaluar la calidad de los documentos ha sido a través de una lectura a fondo de cada uno de los artículos y posteriormente completando el cuadro con los 16 ítems establecidos. Se ha llegado a un acuerdo entre las investigadoras para saber si el artículo cumplía o no el ítem establecido de forma objetiva. Así pues, se puede decir que la media de ITEM cumplidos de los artículos seleccionados para este estudio son de $75.33 \%$.

Por otro lado, Ios análisis adicionales que se han realizado son los ejemplos de servicios web citados en los artículos encontrados, ofreciendo así diversas herramientas para trabajar la actividad física para diversas enfermedades.

\section{RESULTADOS}

\section{Datos seleccionados para el estudio}

Las búsquedas en las diferentes bases de datos mencionadas anteriormente dieron como resultado 126 artículos. Seguidamente se eliminaron los artículos duplicados (98), se eliminaron así mismo por no tener acceso directo (85), solo lo que tuvieron acceso a texto completo (63), se eliminaron además por no cumplir con el idioma seleccionado, por no cumplir el objetivo principal del estudio y por no tener el texto completo (40) y finalmente por no cumplir los criterios de calidad finalmente se seleccionaron un total de 37 referencias. A continuación, se muestra el diagrama de flujo del presente estudio. 
Ilustración 1. Diagrama de flujo. Elaboración propia.

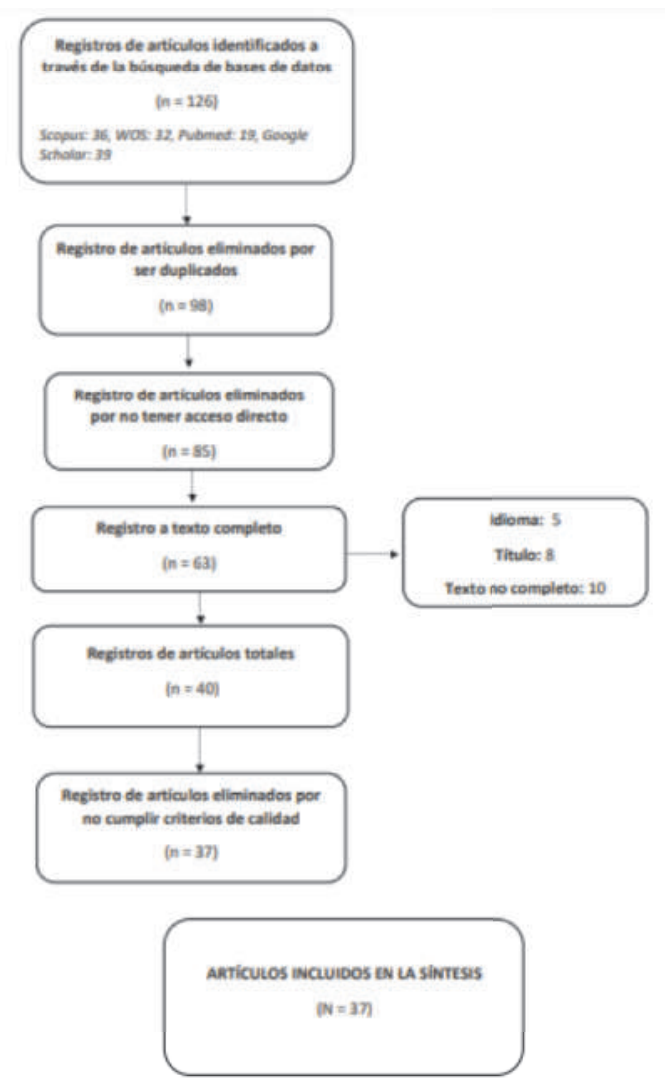

\section{Características de los estudios}

El 35\% de los artículos seleccionados pertenecen a Estados Unidos y un 5,4\% a Australia, Corea del Sur e Italia. El resto pertenecen a otros países distintos y únicamente se ha seleccionado un documento de España.

En cuanto al tipo de método, 9 artículos son cualitativos, 17 son cuantitativos y 11 mixto. La muestra más estudiada dentro de nuestra selección de investigaciones son personas mayores de 18 años, es decir, una población adulta, con 17 estudios que trabajan con dicha muestra. Por otro lado, contamos con 6 estudios con muestras de menores de 18 años, tan solo uno que trabaja con una muestra de mayores de 65 y trece estudios que cuentan con una muestra mixta.

En la gráfica 1, se observa un notorio crecimiento de los estudios en todas las enfermedades encontradas a lo largo de los años, siendo el año 2017 y 2020 los años con más publicaciones sobre las diferentes enfermedades. 
Gráfico 1. Investigaciones a lo largo de los años.

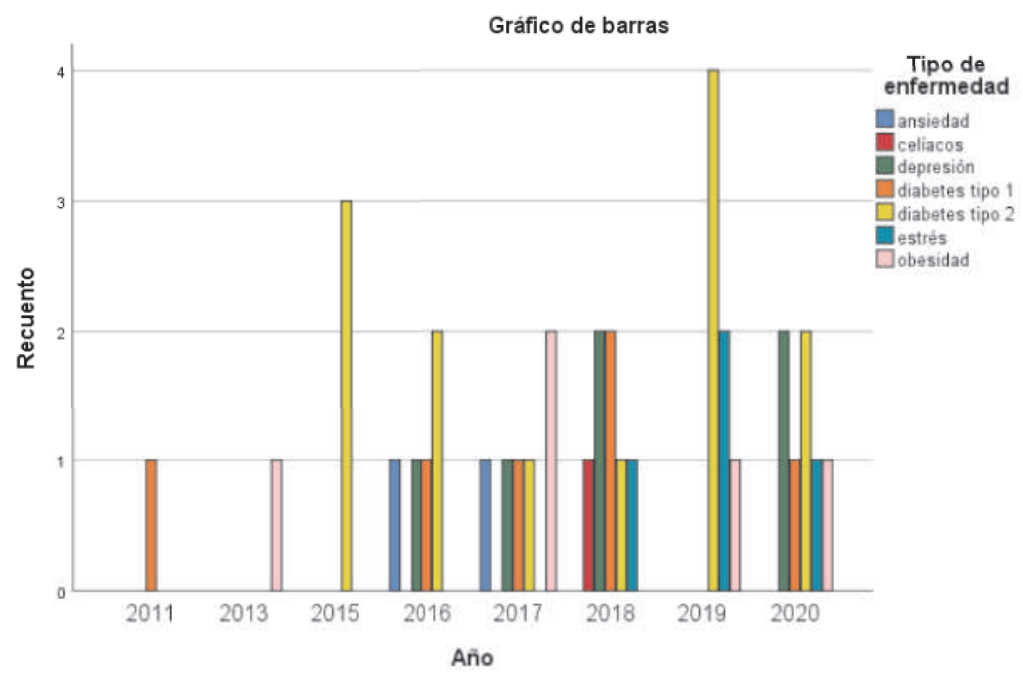

En relación con los tipos de enfermedad, se observa en la gráfica 2 que el mayor número de investigaciones se centran en la diabetes de tipo 2.

Gráfico 2. Tipos de enfermedad.

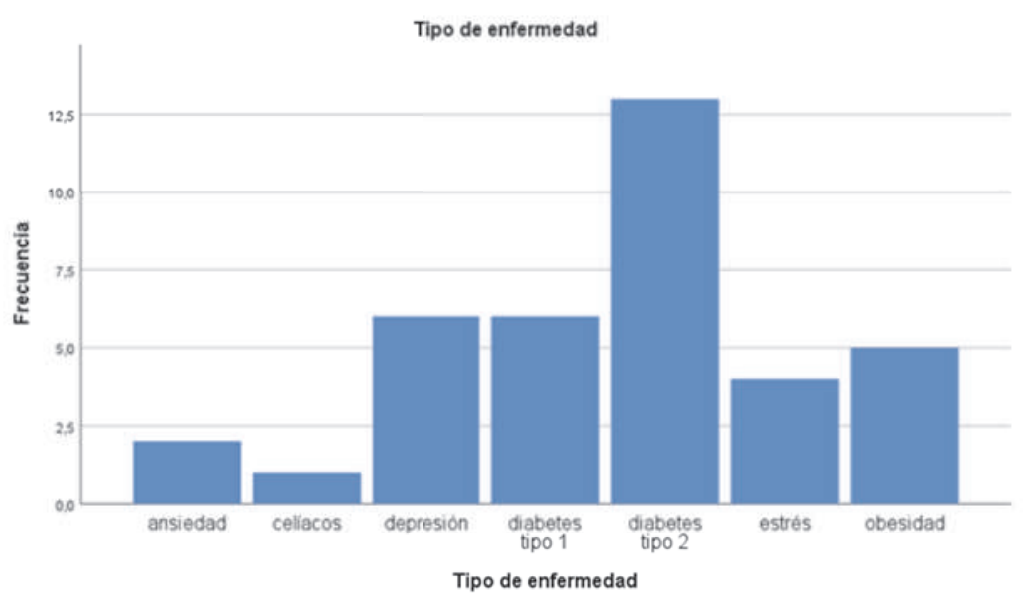

Por otro lado, en el gráfico 3 se muestra los diversos tipos de servicios web que se han encontrado en las investigaciones seleccionadas, siendo las aplicaciones el servicio más estudiado. 
Gráfico 3. Tipos de servicios web.

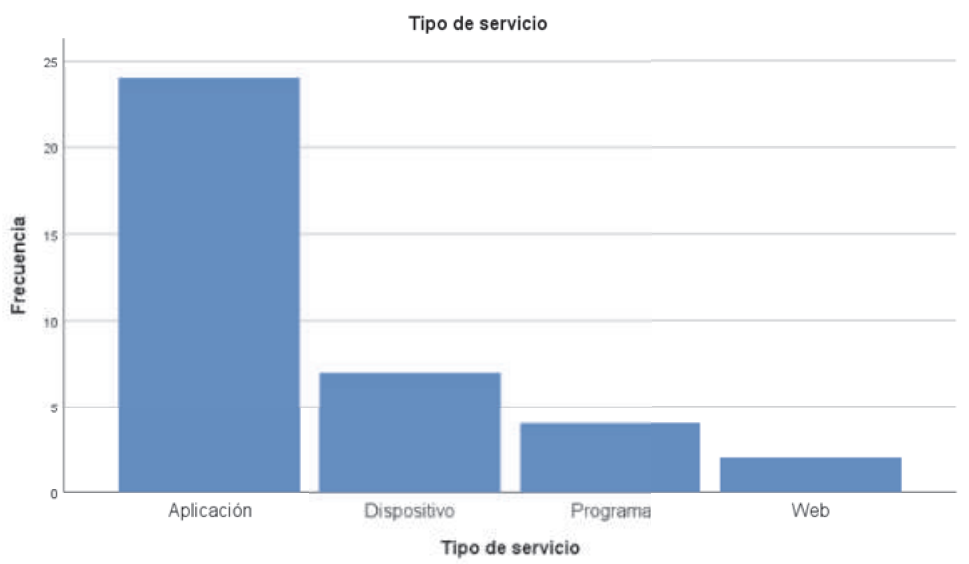

Así pues, se observa en la gráfica 4 la frecuencia del tipo de servicio web según la enfermedad a trabajar, siendo las aplicaciones el servicio más estudiado en las diferentes enfermedades.

Gráfico 4. Tipo de servicio web según la enfermedad.

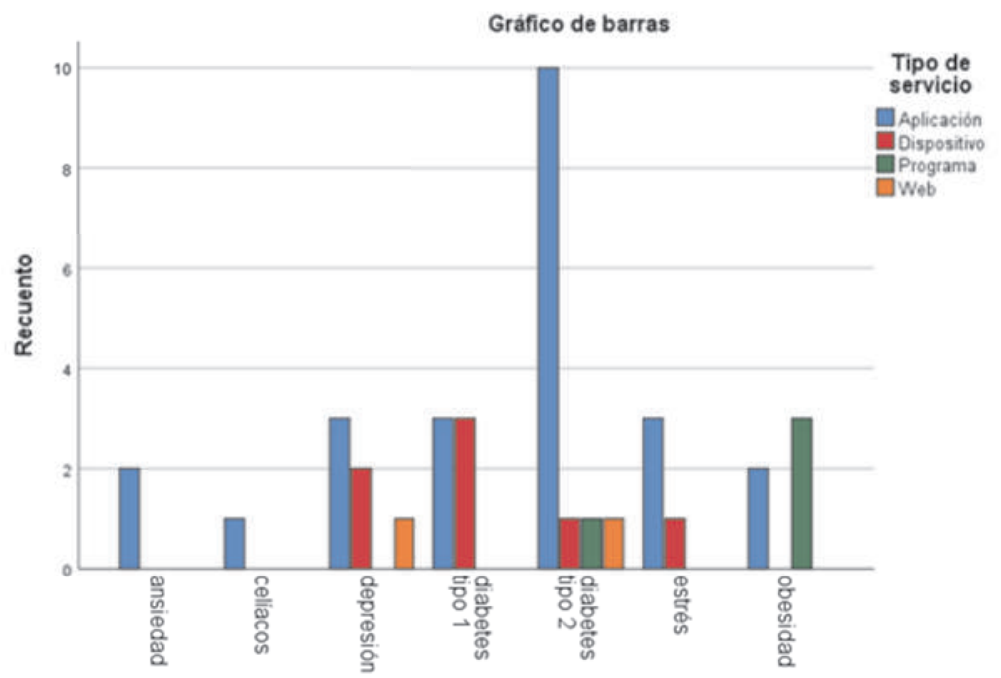

En forma de síntesis, se han estudiado principalmente las variables numéricas y nominales para realizar el análisis descriptivo y la correlación de las variables encontradas, principalmente entre tipo de enfermedad y tipo de servicio web. Las variables de cadena nos han servido para completar el marco teórico, conocer los resultados de estudio, así como de sus beneficios y hallazgos, y conseguir diversas conclusiones basadas en evidencias. 


\section{DISPOSITIVOS O SERVICIOS TECNOLÓGICOS DURANTE LA PRÁCTICA DE ACTIVIDAD FÍSICA PARA PERSONAS CON ENFERMEDAD: REVISIÓN SISTEMÁTICA}

\section{DISCUSIÓN}

El objetivo de este artículo era revisar sistemáticamente artículos publicados desde el año 2013 hasta el 2020 sobre los dispositivos o servicios basados en la tecnología que se han desarrollado para ayudar a los usuarios con discapacidad 0 enfermedades a mantener y crear rutinas relacionadas con la actividad física o deporte. Así como de conocer las diferentes discapacidades o enfermedades que se suelen tratar con este tipo de tecnología.

Hasta la fecha se ha podido observar que ha aumentado la producción científica con respecto a este tema, lo que demuestra cierto interés de la población. Sin embargo, el estudio con los servicios web estudiados resulta algo novedoso por lo que viendo el aumento de dichos estudios se deduce que se publicarán más estudios en los próximos años.

Coincidiendo con Alvárez (2020) y en vista a los resultados que se han obtenido tras esta revisión sistemática, las enfermedades que tienen que ver con el sedentarismo como son las diabetes y/o la obesidad son las más tratadas a través de la tecnología, y que pueden ser controladas a través de estos dispositivos de manera fácil y siendo accesible a la mayoría de las personas como indica Montaña, Amado y Eslava (2014).

Por lo que se observa que la inserción de estas tecnologías en el control de la actividad física proporciona motivación en la que se obtiene como ventajas además el control de enfermedades como la diabetes. (Muños, Villada y Giraldo (2013).

Finalmente, los principales hallazgos encontrados tras la realización de este estudio principalmente es el aumento de la producción científica teniendo como objetivo el estudio de los servicios web para el control de enfermedades. Encontramos además hallazgos como que las aplicaciones son el servicio web más recurrente, la enfermedad más estudiada es la diabetes tipo 2, siguiendo la diabetes tipo 1 como la segunda más estudiada.

Las posibles limitaciones de esta revisión sistemática es que solo se incluyen estudios de tres bases de datos publicados en inglés limitados a publicaciones de acceso abierto por lo que potencialmente podrían pasar por alto otras publicaciones relevantes tanto de acceso cerrado como publicadas en otro idioma.

\section{CONCLUSIONES}

Finalmente, se puede concluir que la producción científica ha aumentado a lo largo de los años en relación con las enfermedades actuales, cumpliendo así, nuestra primera hipótesis.

En cuanto a la segunda hipótesis planteada, "la obesidad es la enfermedad más trabajada en cuanto a los servicios web creados en la actualidad", se rechaza, puesto que la enfermedad más trabajada es la diabetes de tipo 2, según nuestros resultados.

Y, por último, en la tercera hipótesis del trabajo, se confirma tras averiguar que realmente las aplicaciones son el tipo de servicio web que más abunda en la actualidad.

Se afirma así, que los objetivo del presente estudio se cumplen puesto que se se ha realizado una revisión sistemática para conocer los dispositivos o servicios basados en la tecnología que se han desarrollado para ayudar a los usuarios con enfermedades con el objetivo de mantener y crear rutinas relacionadas con la actividad física o deporte. Así como conocer las diferentes enfermedades que se suelen tratar con este tipo de tecnología

De esta manera, se muestra la importancia de fomentar el deporte y ayudar a las personas con limitaciones a su realización para la creación de vidas más saludables. Sin embargo, existen límites que a día de hoy no se ha dado respuesta ni solución. Por ello, como futuros estudios, se propones la investigación de los límites actuales y las posibles soluciones centrados en la diabetes de tipo II.

\section{REFERENCIAS BIBLIOGRÁFICAS}

Álvarez Ávila, F. J. (2020). Promoción del control del sobrepeso y la obesidad mediante el uso de tecnología móvil en la asistencia sanitaria (Trabajo Fin de Máster). Universidad Pública de Navarra, Pamplona (España). Recuperado de: https://hdl.handle.net/2454/37809

Cantúa, J. S., Espinoza, F. A., Flores, J., Romero, M. A. Ruiz, J., y Andrade, P. R. (2018). El marketing deportivo a través medios electrónicos; Estrategia para promover actividad física y disminuir la prevalencia de enfer- 
medades crónicas. Revista de Investigación Académica Sin Frontera: División de Ciencias Económicas y Sociales, (27), 16-16. https://doi.org/10.46589/rdiasf.v0i27.196

Gabrielli, S., Dianti, M., Maimone, R., Betta, M., Filippi, L., Ghezzi, M. \& Forti, S. (2017). Designs of a mobile App for nutrition education (TreC-LifeStyle) and formative evaluation with families of overweight children. JMIR Mhealth Uhealth 5(4). https://mhealth.jmir.org/2017/4/e48

Montaña, A., Amado, C., \& Eslava, H. (2014). Sistemas e-health para el tratamiento de la diabetes. Revista vínculos, 11(2), 111-126. https://doi.org/10.14483/2322939X.9683

Muñoz, E. A., Garrote, D., Sánchez, C. (2017). La Práctica en personas con discpacidad:motivación personal, inclusión y salud. INFAD Revista de psicología, 1 (3), 145-152.

https://www.redalyc.org/pdf/3498/349853537015.pdf

Muñoz, J.E., Villada, J.F., \& Giraldo, J.C., (2013). Exergames: una herramienta tecnológica para la actividad física. Revista Médica de Risaralda,19 (2), 126-130. https://doi.org/10.22517/25395203.8527

Prieto, M. A., (2011). Actividad física y salud. Innovación y experiencia educativa, (42).

${ }^{1}$ Ls ejecución de este artículo está financiado por el Proyecto Erasmus + "AccessCULT - Innovative higher education teaching contents for achieving sustainable: ACCESSibility of CULTural heritage for ALL" (AccessCULT) Grant Agreement number: 2020-1-ES01-KA203-083220 
